\title{
Three-dimensional biomechanical analysis of the bovine humerus
}

\author{
José Benito Bouza-Rodríguez ${ }^{\mathrm{a}, *}$ and Luz Calia Miramontes-Sequeiros ${ }^{\mathrm{b}}$ \\ ${ }^{a}$ School of Industrial Engineering, University of Vigo, Vigo, Spain \\ ${ }^{b}$ Laboratory of Animal Anatomy, Faculty of Biology, University of Vigo, Vigo, Spain
}

\begin{abstract}
There are few reports on the biomechanical analysis of the animal humerus. In this study, a three-dimensional finite element model of the bovine humerus was created, and loaded with the physiological forces acting when the cow is falling or jumping (weight and impact forces). Subsequently the corresponding stress and strain distribution in the humerus for different inclined positions of bone was determined.

The highest stress concentration occurred in the distal humeral diaphysis, both when only the reaction and load transfer forces were considered and when muscle forces were included too, although when muscle forces were included these maximum stresses decreased. In the distal humeral diaphysis, an increase was also observed in the cortical thickness; this may be a bone adaptation to reduce the maximum stresses. By understanding these bone adaptation processes at regional level, non-pharmacological treatments to some bone pathologies could be developed, mainly the ones characterized by loss of bone mass.

Furthermore, taking into account both the humerus fracture strength and the maximum force that muscles can make without breaking, it is deduced that during jumping or falling the cow must maintain the humerus as vertical as possible to better bear the impact. This is in congruity with what was observed.

The interest of this study is in improving the knowledge of animal humerus biomechanics and its application in orthopaedic design and surgical treatments.
\end{abstract}

Keywords: Biomechanical analysis, computer simulation, bone stress distribution, humerus

\section{Introduction}

Human biomechanics has been intensively developed in many fields, from sports to prosthesis and implants design, in contrast with animal biomechanics. Finite element analysis (FEA) is a very common method used to resolve engineering problems, and since 1970 it has also been employed widely to study human bone biomechanics, both in basic investigations and in clinical applications, such as the theory of bone remodelling, muscle and bone interaction, implant design and the evaluation of risk of failure

*Corresponding author: José Benito Bouza-Rodríguez, School of Industrial Engineering, University of Vigo, c/Maxwell s/n, 36310 Vigo, Spain. Tel.: +34 986812217; E-mail: jbouza@uvigo.es.
$[1,6,7,13,21,34,35,41]$. Early investigations were made in two dimensions but later three-dimensional studies have been employed, and one of the most analyzed bones to predict fractures is the human femur $[30,40]$. Furthermore, Crawford et al. [10] showed that the fracture strength prediction made by finite element analysis is better than measurement of the bone mineral density. According to Crawford et al., the attempt to extrapolate from results obtained with human extremities analysis to other animal species is not possible due to the different type of locomotion: bipedal and quadrupedal.

However, there are few basic investigations of stress distribution in animal extremities. In this sense, the dog is one of the most studied animals [8, 33], followed by the horse [25, 36], and the sheep [20]. Moreover, 
most published literature using finite element analysis of animal bones only applied static forces and moments corresponding to the weight bearing of each animal.

Finite element analysis of stress distribution in bovine bones is poorly reported. A few studies have analyzed the bovine tibia [28, 29], but to our knowledge, none of them involved finite element analysis of stress distribution of the bovine humerus. There are no finite element models of this bone with realistic loads, including the muscles forces involved when the animal is standing and in the impact (jumping or falling). Thus, the influence of the muscles on stress distribution in the bovine humerus has not yet been reported.

Furthermore, few papers have described finite element analyses of stress distribution in the humerus of any animal: they investigated one specific area of the human humerus such as the proximal epiphysis [7], the diaphysis [31] or the humero-ulnar joint [26].

Because the humerus is one of the very modified bones during the evolution, the study of this bone is very important in reporting some additional conclusions about its adaptation and evolution.

On the other hand, the natural optimization and adaptation of the bone shape to loading is known as Wolff's law [49]. Wolff and his contemporaries [27] introduced the idea that it is possible to predict the process of bone adaptation and structure by mathematical laws.

It is generally accepted that the mechanical loads that occur during daily activity have an important role in bone maintenance and adaptation, in both the determination of internal structure and external morphology. Many studies have shown the relationship of the adaptation of bone mass and architecture with the strain produced by the environmental loading conditions $[4,5,14,20,25,44,49]$. Bones harmonize with the mechanical strain changes induced by loads, modelling and remodelling the bone tissue to guarantee a suitable morphology and mass of trabecular and cortical bone. As a result of an increase in the loading forces (strong exercises, etc.) an increase in the adult bone mass is observed [44]. In contrast, a reduction in the supported loading forces (disuse, etc.) tends to reduce appositional bone growth and to decrease the adult bone mass $[5,6]$.

Theoretical investigations demonstrated that bone morphology can be predicted using a mathematical theory of bone adaptation to mechanical stress combined with finite element analysis $[4,6]$.
In fact, the lack of literature regarding to stress distribution and bone adaptation in the bovine humerus justifies the need to investigate its biomechanics. It is important not only in the sense to extend the scientific knowledge related to biomechanics of bovine species, but to be able to compare with other animal humerus biomechanics, including human humerus biomechanics, and to be capable of inferring conclusions of interest to improve the scientific knowledge of adaptation and evolution processes and so apply them in prosthesis design or in surgical treatments.

The main objective of this study is to create a three-dimensional finite element model of the bovine humerus, including in the model the physiological forces acting when the cow is jumping or falling (weight and impact forces), and thus determine the consequent stress and strain distribution in this bone for different inclined positions. Another goal of this work is to observe the adaptation processes to stress distribution inside the humerus to elucidate how changes occur in the cortical thickness in each part of the bone.

\section{Materials and methods}

\subsection{Bovine humerus}

The sample was from an abattoir. First the skin and soft tissue were removed, then all the internal liquid was eliminated by drying in the sun for one year. To increase the precision during digitization by a laser scanner, we painted the humerus with a 1:50 dilution of white tempera. This avoided excessive brightness and enhanced the dark areas, in order to get more uniform intensities.

\subsection{Geometrical model}

Three-dimensional geometry of the external surface (Fig. 1) of the humerus was obtained with a threedimensional laser scanner (MINOLTA Vivid 700) based on the laser-triangulation principle.

To increase the scanned model quality the bone was placed in a black box, open at the front. In this way, the scanned model could avoid reflection and atmospheric perturbations, like draughts or powder, which could cross in the laser beam.

Various data captures were made to take into account redundant areas to facilitate the posterior assembly. In the scanning process twenty data captures were 


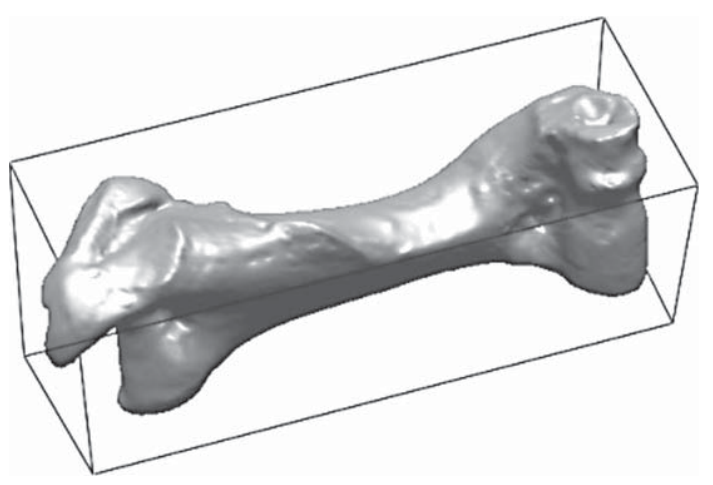

Fig. 1. Scanned humerus model and circumscribed cube to bone.

realized, corresponding to six faces, twelve edges and eight vertices of the cube circumscribed to the bone (Fig. 1). Specific captures were made in the bone areas which contained small details.

In addition, the bone was scanned by computed tomography (CT) to obtain information from the internal layers of the cortical and trabecular bone, resulting in a series of cross sections of the internal humerus. These cross sections were superposed with the previous external surface model, in its exact position.

Subsequently all previous information was treated with Reverse Engineering and Three-dimensional Surface Modeling software (Rhinoceros, Robert McNeel $\&$ Asociates, Washington) obtaining as a result the internal interface surfaces between the cortical and trabecular layers and the medullary cavity. These surfaces were represented by NURBS (non-Uniform Rational B-Splines) [12], which are three-dimensional geometry mathematical representations capable of accurately describing any shape, from the most simple to the most complex organic surfaces. It is a faster and simpler procedure to work with NURBS than with the very large number of points given by the scanner, due to its flexibility, accuracy and easiness of exportation to subsequently deal with other engineering programs such as the finite element ones. The Surface Modeling software provides superior possibilities of 3-D geometry processing (manipulation, treatment, etc.) than the pre-processing module of Finite Element software.

The contour curves (separation curves between each material layer) were obtained from the tomographic sections, and the different internal interface surfaces which define the cortical and trabecular layers and the medullary cavity were achieved by connecting (lofting) the corresponding contour curves (Fig. 2).

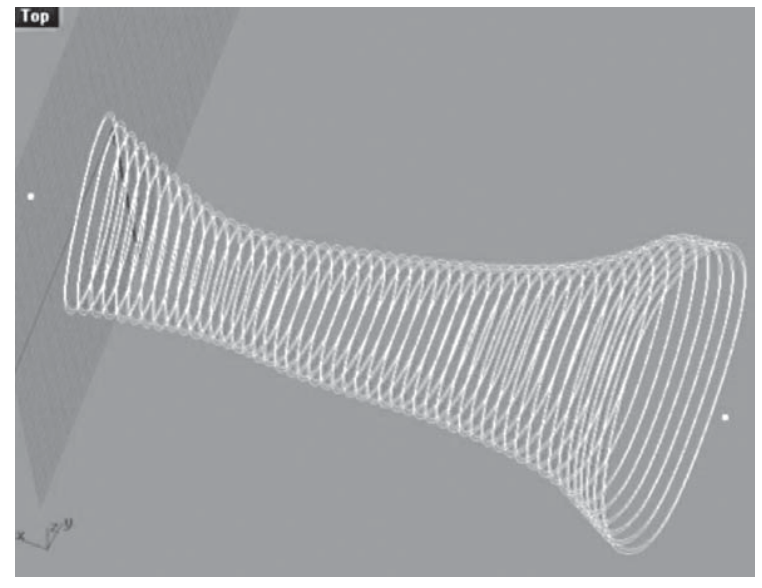

Fig. 2. Corresponding contour curves.

Afterwards all the interface surfaces (external and internal) were exported to the finite element pre-processor, where by means of simple boolean operations, the medullary cavity, cortical and trabecular material layers were obtained, resulting a proper solid model of bone. Figure 3 shows a section of the resulting model.

The bovine humerus modeling was realized taking into account one layer of trabecular bone in the humeral epiphysis, one layer of cortical bone in the humeral mid-diaphysis and lastly the medullary cavity. Figure 3 shows a section of the resulting model.

\subsection{Assignment of mechanical properties}

The finite element model behaviour depends not only on precision of geometrical data of the bone, but a suitable assignment of mechanical properties of bone material to the finite element model; hence this assignation is an essential stage in the bone analyses by finite element method.

The majority of these types of investigations have simplified the problem considering the bone as an isotropic material, but this assumption is correct only if the bone is not subject to significant torsional forces. Since it is widely reported in the literature $[2,32]$ that the cortical and trabecular bone material are orthotropic, recent investigations assigned the orthotropic material properties to bone model [42, 47].

Despite bone behaviour being usually viscoelastic and not lineal, most biomechanical studies consider the bone as an elastic and linear material to simplify the analysis, a supposition reasonably approached to most 


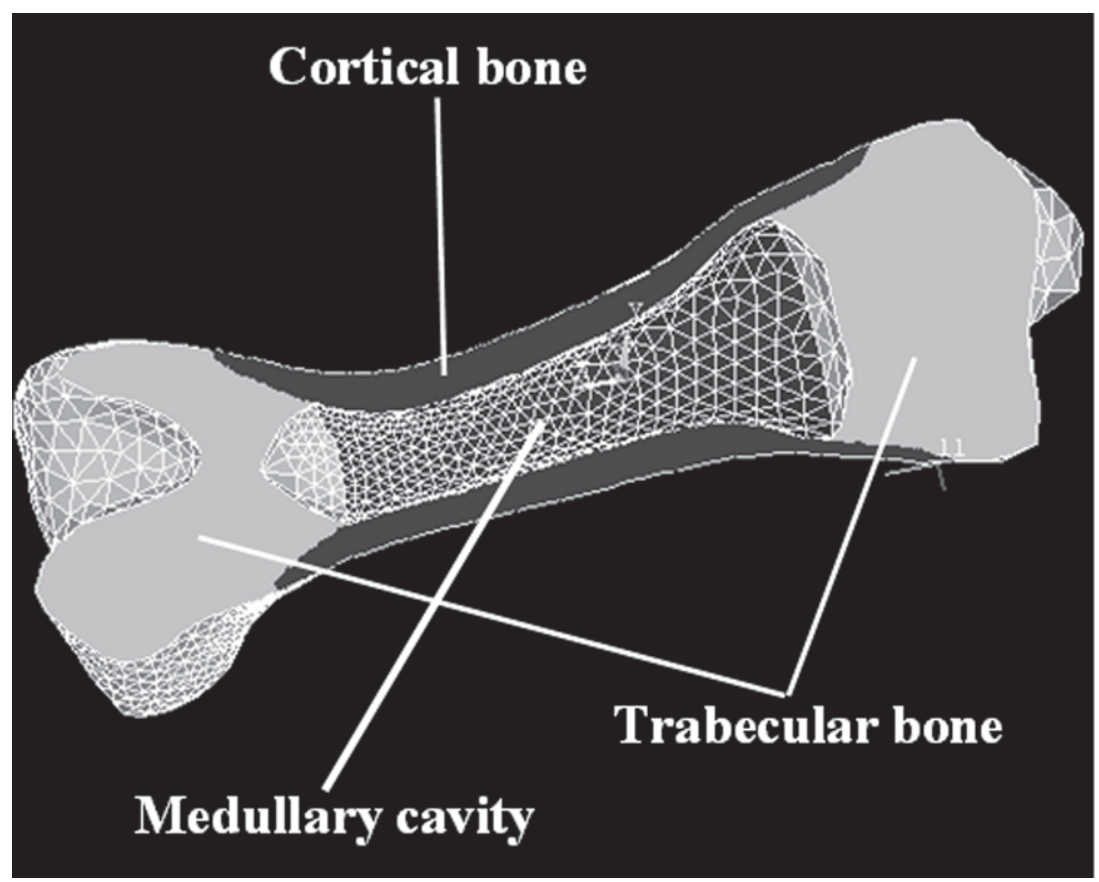

Fig. 3. Longitudinal section of humerus model.

studies of bone stress and strain $[9,32]$. Therefore, the bone behaviour is assumed to be determined by Hooke's law:

$$
[\sigma]=C .[\varepsilon]
$$

where,

$[\sigma]=\left[\sigma_{11} \sigma_{22} \sigma_{33} \sigma_{23} \sigma_{13} \sigma_{12}\right]$ is the stress tensor,

$\mathrm{C}$ is the stiffness matrix, and

$[\varepsilon]=\left[\begin{array}{llllll}\varepsilon_{11} & \varepsilon_{22} & \varepsilon_{33} & \varepsilon_{23} & \varepsilon_{13} & \varepsilon_{12}\end{array}\right]$ is the strain tensor.

The mechanical properties of the bone were assigned separately, on the one hand to the cortical bone layer, and on the other to the trabecular bone layer. Like in the previous finite element analyses [1, 7, 21], we have assigned two material property values, one for cortical and one for cancellous bone.

\subsubsection{Cortical bone material}

The cortical bone of the bovine humerus has a characteristic longitudinal direction defined by the bone axis, which makes the humerus orthotropic with approximately the same stiffness in the perpendicular axes of the transverse plane (transversely isotropic) with a magnitude about 60 per cent of the longitudinal direction one [16]. Therefore, the finite element models that do not take into account the orthotropy of the cortical bone can fail in the generation of accurate results when shear stress is present due to torsion [38] The inclusion by Barker et al. [3] of orthotropic cortical bone properties improves significantly resultant values from finite element model when torsion is considered. Thus, we decided to consider the cortical bone as a transversely isotropic material.

The mechanical properties of the bovine cortical bone were obtained from the literature, mainly from Kohles's ultrasonic experiment results [19]. The values assigned were: Young's modulus $\left(\mathrm{E}_{1}=22 \mathrm{GPa}\right.$, $\left.\mathrm{E}_{2}=11.3 \mathrm{GPa}, \mathrm{E}_{3}=11.3 \mathrm{GPa}\right)$, and shear modulus $\left(\mathrm{G}_{12}=5.4 \mathrm{GPa}, \mathrm{G}_{13}=5.4 \mathrm{GPa}, \mathrm{G}_{23}=3.8 \mathrm{GPa}\right)$. Where the subindex 1 corresponds with the longitudinal bone axis and the subindexes 2 and 3 corresponds with the two orthogonal axes in the transverse plane.

\subsubsection{Trabecular bone material}

Trabecular bone is more complex than compact bone. As in the previous case, we have applied in this study the consideration of trabecular bone tissue as a transversely isotropic material to describe the material behaviour according to the direction.

The mechanical properties were obtained from the literature of trabecular bovine bone, mainly from values obtained by Turner et al. [43], assigning the following values: Young's modulus $\left(\mathrm{E}_{1}=2.9 \mathrm{GPa}\right.$, 


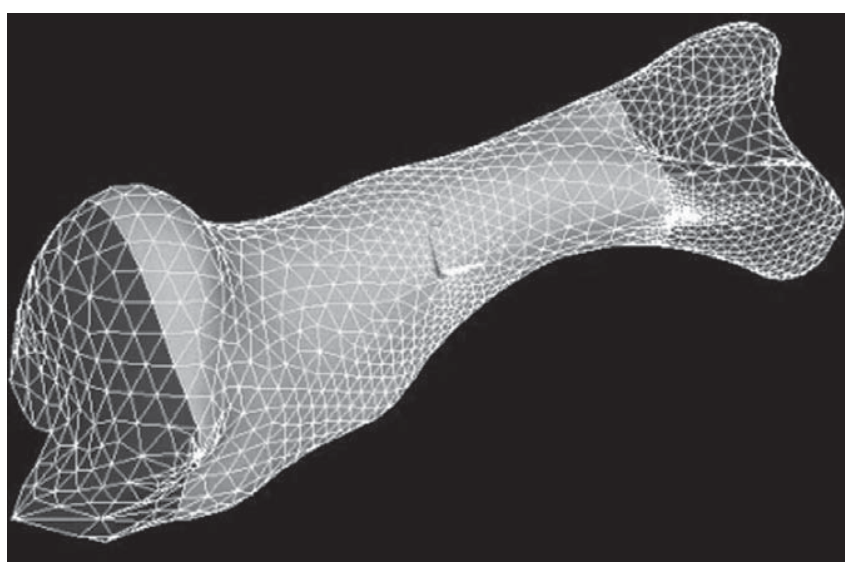

Fig. 4. External contour of finite element resultant mesh.

$\left.\mathrm{E}_{2}=0.4 \mathrm{GPa}, \mathrm{E}_{3}=0.4 \mathrm{GPa}\right)$, and shear modulus $\left(\mathrm{G}_{12}=130 \mathrm{MPa}, \mathrm{G}_{13}=130 \mathrm{MPa}, \mathrm{G}_{23}=80 \mathrm{MPa}\right)$. Where the subindexes 1,2 , and 3 have the same meaning as in the previous case.

\subsection{Mesh generation}

The solid model previously created was automatically meshed with 10-node tetrahedral elements, according to investigations [45] that reported more accurate results with this element type in the solid models of the bone, since these elements have quadratic displacement behaviour and have the ability to fit well to irregular geometry models like in the bone case. The element has three freedom degrees in each node: translation in $\mathrm{X}, \mathrm{Y}, \mathrm{Z}$ directions, and it has hyperelasticity, mechanical tenacity, high deflection and deformation. The resultant bovine humerus mesh contains 29055 elements with 46071 nodes (see Fig. 4).

\subsection{Boundary conditions and loading simulation}

The model was subjected to various loading and boundary conditions. First we carried out basic engineering simulations and then we made more complete simulations taking into account the muscle forces, to make them more like the real-life situation.

\subsubsection{Basic simulations}

We made the following basic simulations: a) Compression test, b) Bending test, c) Torsion test, and d) Combined test of the three previous ones ( $a, b$ and $c$ ).
The basic simulations were carried out under a displacement constraint at one end of the bone model and subjecting under loading at the opposite end.

In the compression test, the bovine humerus model was loaded with a force of $28540 \mathrm{~N}$. This value was selected in order to be able to compare this basic simulation with the next test: a complete simulation with the muscle forces (see Table 2). This force corresponds approximately to an increase of 14 times the weight that supports the leg of a cow of $850 \mathrm{~kg}$ during weight bearing. This ratio is given by:

$$
\frac{28540 \mathrm{~N}}{\left(\frac{850 \mathrm{~kg} \times 9.8 \mathrm{~N} / \mathrm{kg}}{4 \operatorname{legs}}\right)}=13.7 \text { times } \approx 14 \text { times }
$$

It is known that the impact forces are much more elevated than the animal weight bearing (it is deduced from mechanical equations), thus with this 14 times superior load than the normal weight it becomes possible to simulate a representative jump (impact), in this case assuming that the cow falls down on its four limbs without folding its knees, or, effectively the same thing, that the impact is absorbed by limb bone compression.

Also in the bending test a load of $28540 \mathrm{~N}$ was applied, and in the torsion test a torque of two forces of $10000 \mathrm{~N}$ was applied. In the combined test all previous loads were overlapped.

\subsubsection{Complete simulation with the muscle forces}

Several simulations with different bovine humerus positions were made, taking into account weight and impact forces transmitted to the bone as if they were 


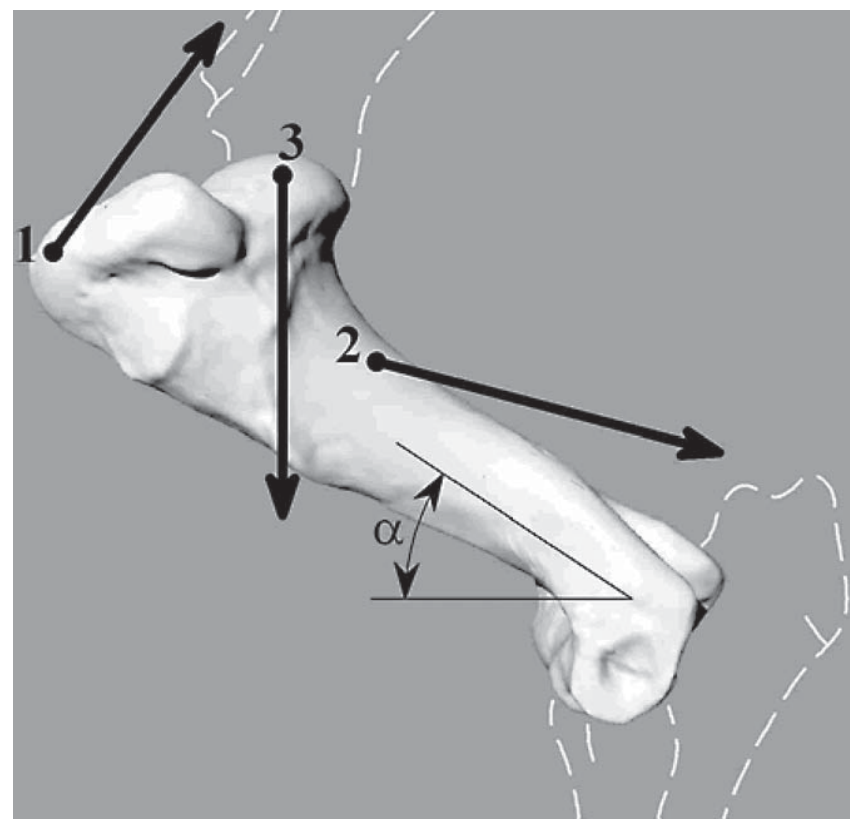

Fig. 5. Considered forces acting on humerus, with their application points and action directions. (1) Total force carried out by supraspinatus, infraspinatus, pectoralis and subscapularis muscles. (2) Triceps brachialis muscle force. (3) Cow weight plus impact force. ( $\alpha$ ) Angle formed by the axis of the humerus with the horizontal plane. Dashed lines represent the adjacent bone ends, scapula and radio-ulna.

the maximum forces that can be developed in each moment by muscles inserted in the humerus.

Figure 5 represents the muscle forces, with their application points and actuation directions, which were used in each complete simulation. Only muscles involved with falling were studied, since they are the ones that carry out the effort to avoid the abrupt bone movement to cushion the impact. All muscles with near application points and similar actuation directions were gathered, therefore in Fig. 5 vector (1) represents the total force performed by supraspinatus, infraspinatus, pectoralis and subscapularis muscles, vector (2) represents the triceps brachialis, and vector (3) represents the weight of the cow plus the impact force during jumping or falling. The displacement constraint (in translation) was made at the distal end, in the radioulnar joint, just allowing rotation.

Once all muscles that perform the falling effort were identified, the maximum forces that these muscles could develop in the bone before their failure were calculated. According to experimental results, the maximum muscle force depends on muscle crosssectional area [46, 48]; for humans it is about $35 \mathrm{~N} / \mathrm{cm}^{2}$. If we compare the different data obtained by several authors $[9,24]$, the average relationship between human and bovine mechanical properties is nearly $15 \%$
Table 1

Forces obtained for each muscle and for each muscle group considered, in function of its own section

\begin{tabular}{lccc}
\hline Muscle & Area & Force & Total force in the direction \\
\hline Supraspinatus & $40 \mathrm{~cm}^{2}$ & $1600 \mathrm{~N}$ & (1) $6750 \mathrm{~N}$ \\
Infraspinatus & $65.8 \mathrm{~cm}^{2}$ & $2630 \mathrm{~N}$ & \\
Pectoralis & $35 \mathrm{~cm}^{2}$ & $1400 \mathrm{~N}$ & \\
Subscapularis & $28 \mathrm{~cm}^{2}$ & $1120 \mathrm{~N}$ & \\
Triceps Brachialis & $118.12 \mathrm{~cm}^{2}$ & $4725 \mathrm{~N}$ & (2) $4725 \mathrm{~N}$ \\
\hline
\end{tabular}

(1) Direction of force 1 and (2) direction of force 2, as Fig. 5.

higher for the bovine; that is why we elected to use a maximum stress of $40 \mathrm{~N} / \mathrm{cm}^{2}$ for the bovine muscle. In Table 1 the forces obtained for each muscle and for each group of muscles, in function of its sections, are shown.

Subsequently, the maximum force (3) supported by the bovine humerus during jumping or falling without muscle failure was calculated in four different $\alpha$ positions: $\alpha=75^{\circ} ; \alpha=45^{\circ} ; \alpha=15^{\circ}$ and $\alpha=0^{\circ}$; where $\alpha$ is the angle of the humerus axis with the horizontal plane (see Fig. 5).

Applying the mechanical equations $\left(\sum\right.$ Forces $=0$ and $\sum$ Moments $=0$ ) for each of the four cases in the force system of Fig. 5 with the values of Table 1, the results shown in Table 2 were obtained for the force (3). These equations are: 
Force (1) + Force (2) + Force (3) + Reaction (on radioulnar joint $)=0$

Moment(of force(1) about radio-ulnar joint) + Moment(of force(2) about radio-ulnar joint) + Moment (of force(3) about radio-ulnar joint) $=0$

The finite element analyses were carried out with force values of Tables 1 and 2, for each different case.

\section{Results}

In evaluating the maximum stress, the calculated stresses in some areas were ignored, namely: the stresses where the loads were applied and the one where the constraints were attached; since the value of the stresses calculated by the system at these points is not reliable due to their fictitious accumulation. This means the peak stresses that appear in the distal epiphysis of the bovine humerus on the radio-ulnar joint

\section{Table 2}

Values of maximum force (3) that bone can support during falling or jumping without muscle failure, in four different $\alpha$ positions, where $\alpha$ is the angle formed by the axis of the humerus and the horizontal plane, see Fig. 5

\begin{tabular}{lc}
\hline Angle $(\alpha)$ & Force (3) \\
\hline $75^{\circ}$ & $28540 \mathrm{~N}$ \\
$45^{\circ}$ & $12869 \mathrm{~N}$ \\
$15^{\circ}$ & $8112 \mathrm{~N}$ \\
$0^{\circ}$ & $6061 \mathrm{~N}$ \\
\hline
\end{tabular}

were not considered (red colour in the figures), because they are caused by the displacement constraint.

\subsection{Basic simulations}

For all the basic simulations performed, the maximum stress was reached in the distal diaphysis of the bovine humerus (Figs. 6 and 7).

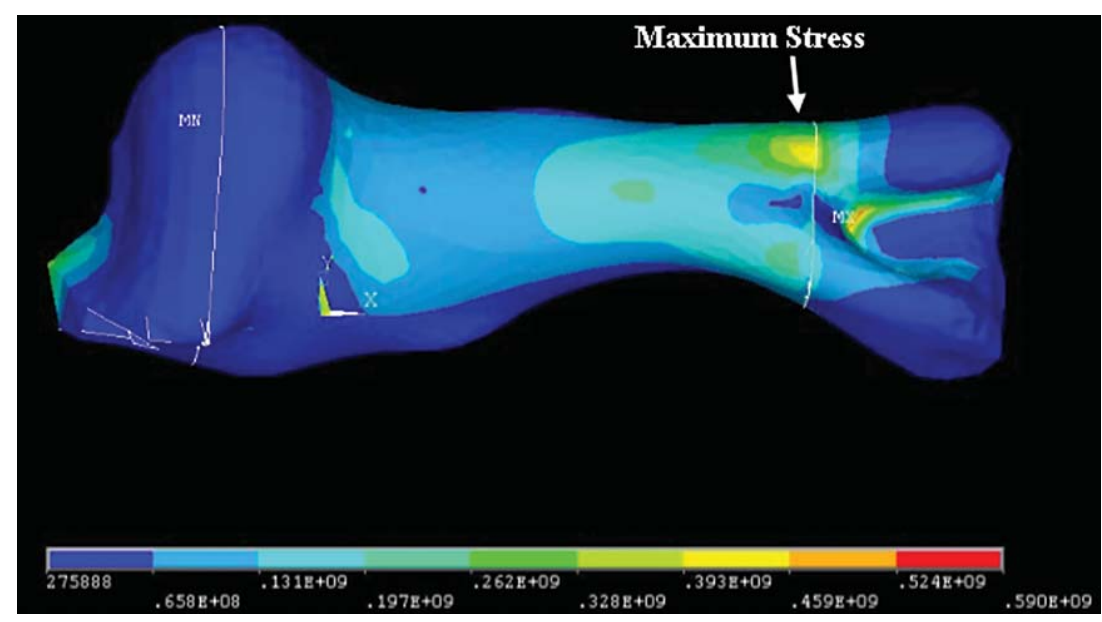

Fig. 6. Stress distribution in basic compression test

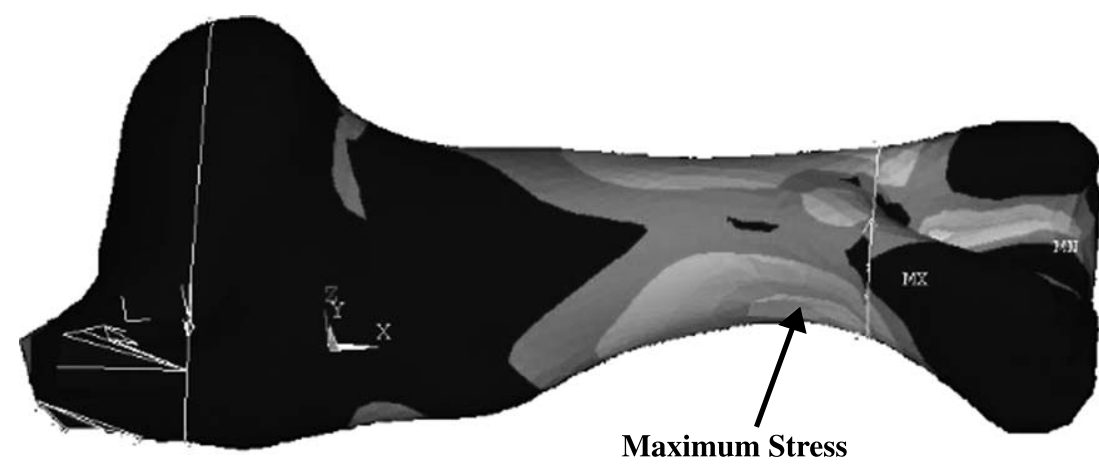

Fig. 7. Stress distribution in basic bending test. 


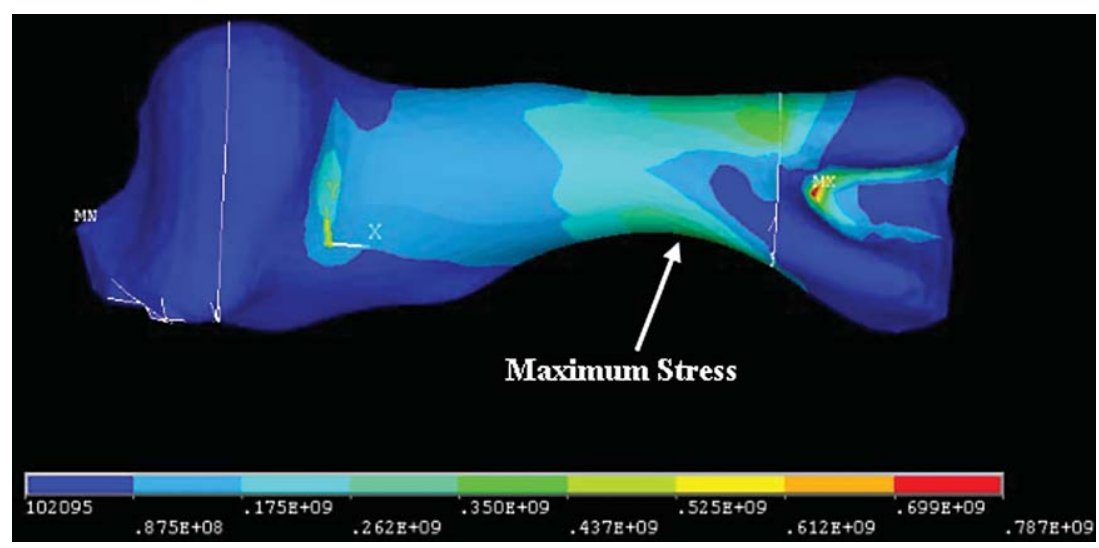

Fig. 8. Stress distribution in complete test with the muscle forces considered, when the humerus was forming an angle $\alpha$ of $75^{\circ}$ with the horizontal plane (see Fig. 5).

The maximum stress reached in the compression test was $393 \mathrm{MPa}$ (Fig. 6), in the bending test the maximum value of stress was $960 \mathrm{MPa}$ (Fig. 7), the maximum value reached in the torsion test was $223 \mathrm{MPa}$, and lastly, in the combined test the maximal value reached was $1290 \mathrm{MPa}$.

\subsection{Complete simulation with the muscle forces}

In all these loading cases, the peak stress was reached at the distal diaphysis of the bovine humerus (Fig. 8), like in the previous basic cases. The highest stress values were reached when the humerus was forming an angle $\alpha$ of $75^{\circ}$ with the horizontal plane (Fig. 5), since in this position it was possible to apply the highest loadings without breaking the muscles (see Table 2); and the maximum stress value of the humerus was $350 \mathrm{MPa}$ (Fig. 8). In the other loading positions it is not possible to reach this maximum stress value because, before this happens, the muscles break. So when the humerus is forming an angle $\alpha$ of $45^{\circ}$ with the horizontal plane, the maximum stress value reached was $158 \mathrm{MPa}$, when the angle $\alpha$ was $15^{\circ}$ the maximum stress value reached was $120 \mathrm{MPa}$ and when $\alpha$ was $0^{\circ}$ the maximum stress value reached was $110 \mathrm{MPa}$ (see Table 3 ). Note that the values of this table correspond to critical loading conditions.

\section{Discussion}

The bovine humerus was internally and externally modeled with its corresponding layers, subsequently, the three-dimensional finite element analysis of the stress distribution in the humerus subjected to external
Table 3

Maximum stress reached in the complete test, in four different $\alpha$ positions, where $\alpha$ is the angle of the humerus axis with the horizontal plane, and force (3) is the force applied during falling or jumping without muscles failure, see Fig. 5

\begin{tabular}{lcc}
\hline Angle $(\alpha)$ & Force $(3)$ & Maximum stress \\
\hline $75^{\circ}$ & $28540 \mathrm{~N}$ & $350 \mathrm{MPa}$ \\
$45^{\circ}$ & $12869 \mathrm{~N}$ & $158 \mathrm{MPa}$ \\
$15^{\circ}$ & $8112 \mathrm{~N}$ & $120 \mathrm{MPa}$ \\
$0^{\circ}$ & $6061 \mathrm{~N}$ & $110 \mathrm{MPa}$ \\
\hline
\end{tabular}

loads was carried out. During modeling, the humerus was divided in two different kinds of materials (cortical and trabecular bone) since these two materials have a different behaviour when subjected to the same loading conditions.

The results of these finite element simulations show that if the muscle forces are included in the complete simulation the maximum stresses decrease (350 MPa), specially if compared with the basic bending test (960 $\mathrm{MPa}$ ), when the loads applied were of a similar order of magnitude. The basic simulations only consider the radio-ulnar joint reactions and different cases of load transfer from the scapula to the humerus, but with the muscle insertions in the complete simulation we obtain an accurate approach to the real biologic system. Once more, we can see here how the biology optimizes its systems, in this case the muscles aid to avoid bone fractures, decreasing and distributing the maximum stresses. This observation agrees well with other works $[33,41]$ reporting that the internal stresses result in a decrease if the muscles forces are taken into account in the bone model, in comparison with when only the reaction forces are considered. 


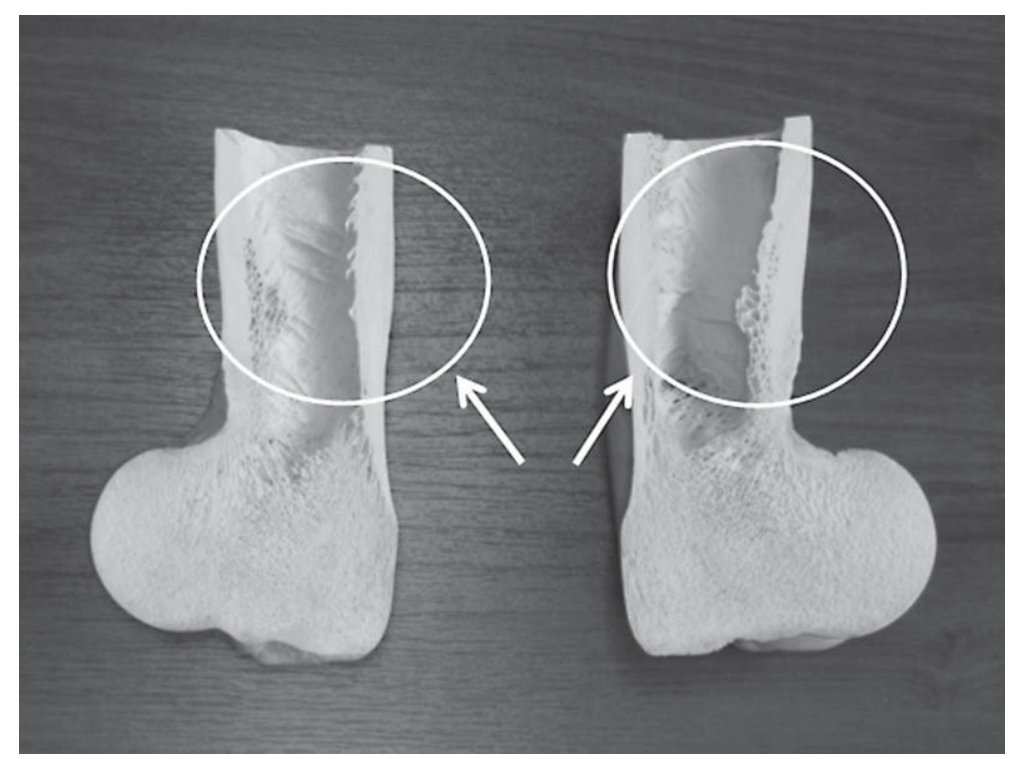

Fig. 9. Longitudinal section of distal part of the humerus (medial and lateral half) showing the area with the highest cortical layer thickness.

Moreover, this study found out that the cortical bone layer suffers the highest mechanical efforts, and when changing from the cortical to the trabecular bone layer, the stresses appreciably decrease. This finding is in congruence with another finite element analysis of human humerus [7].

As Figs. 6, 7 and 8 show, the highest stress concentration occurs in the distal humeral diaphysis, which is the zone with the lower section of the bone, and where there is a change in the bone material. This stress concentration zone will be the most susceptible area to fractures when the bone is overloaded.

From the sagittal section photography (see Fig. 9) it is observed that this is a zone that has suffered an increase in its cortical thickness. This observation suggests that there is a bone adaptation to minimize the maximum stresses generated in this area. Thus, it is noticed that the bone, which is part of a living organism, undergoes the remodelling process to strengthen the zones where high stresses are produced, to minimize them and better adapt to the mechanical efforts that are seen during animal life.

Other reports have already suggested that regional variations in cortical bone morphology are more obvious in the diaphysis of limb bones that in normal conditions are subject to bending [37], due to, in this case, there are more stress differences between the bone zones. Other works have found that mechanical loads on the skeleton during growth can increase the cross section of the bone diaphysis [15, 18]. For example, the case of the professional tennis players where an increase in the cortical thickness (about 32 per cent) of the dominant arm in comparison with the non dominant arm was found [17]. Bone skeleton adapted to high loads, augmenting the cortical thickness by depositional growth to reduce local stress [23] in order to decrease the stress fracture risk. The relationship between the cortical thickness of the femoral neck and the weight of the animal, in the human case, is characterized by a diminution in the cortical thickness of the superior zone related to the inferior zone of the femoral neck, because superior zone is hardly subject to stress since the abductor muscle neutralizes the tensile stress produced by animal weight that should be acting in this area. In contrast, no significant reduction was found in the cortical thickness of the superior zone of the femoral neck in other primate species lacking an effective abductor muscle [22].

Our results are in accordance with Wolff's law [49] once more, in this case in the bovine humerus, employing the FEA technology. Also, they ratify the idea that bones are designed to have a minimum mass to perform specific functions [11], since bones have an increased thickness only where it is necessary to avoid fracture risk. However, these observations are not in congruence with some finite element analysis of human bones [41] where, although there were differences in the bone stress distribution at studied zones, the corresponding 
cortical thickness variations as a possible adaptation, according to Wolff's law [49], were not found.

By understanding these bone adaptation processes at regional level, non-pharmacological treatments to some bone pathologies could be developed, mainly the ones characterized by loss of bone mass.

Taking into account this finding, another possible investigation could be the comparison between increased zones of different bovine humerus to evaluate if these zones are due to evolution. For this, we recommend taking the sagittal section shown in Fig. 9, and comparing the area inside the circle in this figure.

Furthermore, the compressive ultimate stress in the bovine cortical bone can reach $237 \mathrm{MPa}$ [24]. Thus it is deduced that if the animal, during falling or jumping, maintains its humerus as vertical as possible $\left(\alpha=75^{\circ}\right.$ in Fig. 5) and the impact force is so high as to break the bone, the bovine humerus would fracture since the muscles would support it. In this case with a force (3) of $28540 \mathrm{~N}$ the maximum stress reached is $350 \mathrm{MPa}$ (see Table 3), and the humerus would fracture in the distal zone of its diaphysis, while its muscles would not break, as was shown in a previous section. This force of $28540 \mathrm{~N}$ represents an impact equivalent to fourteen times the animal weight $(850 \mathrm{~kg})$, as was previously seen.

In contrast, if the animal folds its knees during falling or jumping $\left(\alpha=45^{\circ}, 15^{\circ}, 0^{\circ}\right.$ in Fig. 5) the ultimate stress in the bovine humerus is not reached (see Table 3) because muscles break before this happens (see previous sections); that is, in this case the bone would not fracture but rather the muscles would break. But, as shown in Table 2, with a force (3) of $6061 \mathrm{~N}$ in the most unfavorable case, it is only possible to support an impact force three times equivalent to the weight of the cow, or a non significant jump.

Since the distribution and direction of the muscle forces and of the weight in the humerus, the force of the muscles is more effective when the humerus is as vertical as possible $\left(\alpha=75^{\circ}\right)$, due to mechanical laws (principle of leverage).

Hence, it is deduced that when a cow or a bull is jumping or during running stumbles and falls down, the animal must maintain the humerus in the most vertical position as possible, since this is the position in which more impact bears, and it must prevent the humerus from coming to more horizontal positions $\left(\alpha=15^{\circ}\right.$, $\alpha=0^{\circ}$, in Fig. 5), because the bone in these positions cannot bear more than the animal's own weight. Such has been observed by us: the bovines pitch forward in a jump if the humerus folding is severe. In bullfights, even when the bull is facing the toreador and gives small jumps with its front half, it does not fold its forelimbs, maintaining them very firm and stretched during falling: the bull absorbs the impact of the jump by compression of the forelimb bones (humerus and others), and not folding its knees.

Another application of the 3D model of the humerus developed is to perform a dynamic analysis of the bone group of the cow forelimbs, in conjunction with other bone models (radius, ulna, metacarpal, phalanx, scapula, ... ), a subject that is being investigated by us.

\section{Conclusions}

In this work, a three-dimensional finite element model of the cow humerus was created, with anatomical accuracy, loaded by physiological forces implicated when the animal jumps or falls down (weight and impact forces), and then the consequent stress and strain distributions in this bone were determined. The three-dimensional finite element analysis of the bovine humerus provides previously unavailable information that could be used by biologists to improve our knowledge of the adaptation and evolution processes, and for veterinarians or investigators of orthopaedics who want to use the cow as a model. This study likewise constitutes the basis for future work on the biomechanics of bovine humerus. Also in this investigation the adaptation processes to the stress distribution produced inside the humerus were elucidated: an examination of the cortical thickness in function of the stress on each area of the bone.

\section{Acknowledgments}

We are grateful to Professor Mark Davies (University of Sunderland) for his critically reading and helpful comments and to Ricardo Fernández and Antonio Palanca (University of Vigo) for their help in this study.

\section{References}

[1] K. Akca and M.C. Cehreli, Biomechanical consequences of progressive marginal bone loss around oral implants: A finite element stress analysis, Medical and Biological Engineering and Computing 44 (2006), 527-535. 
[2] R.B. Ashman, J.Y. Rho and C.H. Turner, Anatomical variation of orthotropic elastic moduli of the proximal human tibia, Journal of Biomechanics 22 (1989), 895-900.

[3] D.S. Barker, D.J. Netherway, J. Krishnan and T.C. Hearn, Validation of a finite element model of the human metacarpal, Medical Engineering and Physics 27 (2005), 103-113.

[4] G.S. Beaupré, T.E. Orr and D.R. Carter, An approach for time-dependent modeling and remodeling-application: A preliminary remodeling simulation, Journal of Orthopaedic Research 8 (1990), 662-670.

[5] D.D. Bikle, T. Sakata and B.P. Halloran, The impact of skeletal unloading on bone formation, Gravitational and Space Biology Bulletin 16 (2003), 45-54.

[6] D.R. Carter, M.C. van der Meulen and G.S. Beaupré, Mechanical factors in bone growth and development, Bone 18 (1996), 5-10.

[7] P. Clavert, M. Zerah, J. Krier, P. Mille, J.F. Kempf and J.L. Kahn, Finite element analysis of the strain distribution in the humeral head tubercles during abduction: Comparison of young and osteoporotic bone, Surgical and Radiologic Anatomy 28 (2006), 581-587.

[8] J.C. Coleman, R.T. Hart, I. Owan, Y. Tankano and D.B. Burr, Characterization of dynamic three-dimensional strain fields in the canine radius, Journal of Biomechanics 35 (2002), 1677-1683

[9] S.C. Cowin, Bone Mechanics Handbook, Second Ed., CRC Press, Boca Raton, FL, 2001.

[10] R.P. Crawford, C.E. Cann and T.M. Keaveny, Finite element models predict in vitro vertebral body compressive strength better than quantitative computed tomography, Bone 33 (2003), 744-750.

[11] J.D. Currey and R.M. Alexander, The thickness of the walls of tubular bones, Journal of Zoology 206 (1985), 453-468.

[12] G. Farin, Curves and Surfaces for Computer Aided Geometric Design, Fourth Ed., Academic Press, San Diego, CA, 1997.

[13] R.J. Frayne, M. Schwartz and J.P. Dickey, A mastication mechanism designed for testing temporomandibular joint implants, Applied Bionics and Biomechanics 9 (2012), 241-247.

[14] H. Frost, Bone's mechanostat: A 2003 update. Anatomical Record - Part A Discoveries in Molecular, Cellular, and Evolutionary Biology 275 (2003), 1081-1101.

[15] H. Haapasalo, S. Kontulainen, H. Sievanen, P. Kannus, M. Jarvinen and I. Vuori, Exercise-induced bone gain is due to enlargement in bone size without a change in volumetric bone density: A peripheral quantitative computed tomography study of the upper arms of male tennis players, Bone $\mathbf{2 7}$ (2000), 351-357.

[16] P.F. Hubsch, J. Middleton, E.A. Meroi and A.N. Natali, Adaptive finite-element approach for analysis of bone/prosthesis interaction, Medical and Biological Engineering and Computing 33 (1995), 33-37.

[17] H.H. Jones, J.D. Priest, W.C. Hayes, C.C. Tichenor and D.A. Nagel, Humeral hypertrophy in response to exercise, Journal of Bone and Joint Surgery-American 59 (1977), 204-208.

[18] P. Kannus, H. Sievanen and I. Vuori, Physical loading, exercise, and bone, Bone 18 (1996), 1S-3S

[19] S.S. Kohles, Applications of an anisotropic parameter to cortical bone, Journal of Materials Science: Materials in Medicine 11 (2000), 261-265.
[20] L.E. Lanyon and S. Bourne, The influence of mechanical function on the development and remodeling of the tibia. An experimental study in sheep, Journal of Bone and Joint Surgery-American 61 (1979), 263-273.

[21] S.L. Lin, S.Y. Lee, L.Y. Lee, W.T. Chiu, C.T. Lin and H.M. Huang, Vibrational analysis of mandible trauma: Experimental and numerical approaches, Medical and Biological Engineering and Computing 44 (2006), 785-792.

[22] C.O. Lovejoy, The natural history of human gait and posture: Part 2. Hip and thigh, Gait and Posture 21 (2005), 113-124.

[23] R.B. Martin, Mathematical model for repair of fatigue damage and stress fracture in osteonal bone, Journal of Orthopaedic Research 13 (1995), 309-316.

[24] R.B. Martin, D.B. Burr and N.A. Sharkey, Skeletal Tissue Mechanics, Springer-Verlarg, New York, 1998.

[25] M.W. Mason, J.G. Skedros and R.D. Bloebaum, Evidence of strain-mode-related cortical adaptation in the diaphysis of the horse radius, Bone 17 (1995), 229-237.

[26] B. Merz, F. Eckstein, S. Hillebrand and R. Putz, Mechanical implications of humero-ulnar incongruity — Finite element analysis and experiment, Journal of Biomechanics 30 (1997), 713-721.

[27] G.H. Meyer, Die architektur der spongiosa. Archiv für Anatomie, Physiologie und Wissenschaftliche Medizin 34 (1867), 615-625.

[28] E.F. Morgan, H.H. Bayraktar, O.C. Yeh, S. Majumdar, A. Burghardt and T.M. Keaveny, Contribution of intersite variations in architecture to trabecular bone apparent yield strains, Journal of Biomechanics 37 (2004), 14131420.

[29] S. Nagaraja, T.L. Couse and R.E. Guldberg, Trabecular bone microdamage and microstructural stresses under uniaxial compression, Journal of Biomechanics 38 (2005), 707-716.

[30] T. Ota, I. Yamamoto and R. Morita, Fracture simulation of femoral bone using finite-element method: How a fracture initiates and proceeds, Journal of Bone and Mineral Metabolism 17 (1999), 108-112.

[31] D. Plausinis, C. Greaves, W.D. Regan and T.R. Oxland, Ipsilateral shoulder and elbow replacements: On the risk of periprosthetic fracture, Clinical Biomechanics 20 (2005), 1055-1063.

[32] J.Y. Rho, An ultrasonic method for measuring the elastic properties of human tibial cortical and cancellous bone, Ultrasonics 34 (1996), 777-783.

[33] R. Shahar, L. Banks-Sills and R. Eliasy, Stress and strain distribution in the intact canine femur: Finite element analysis, Medical Engineering and Physics 25 (2003), 387-395.

[34] K. Shahim, J.M. Drezet, J.F. Molinari, R. Sinkus and S. Momjian, Finite element analysis of normal pressure hydrocephalus: Influence of CSF content and anisotropy in permeability, Applied Bionics and Biomechanics 7 (2010), 187-197.

[35] S. Sivarasu and L. Mathew, Finite -element-based design optimisation of a novel high flexion knee used in total knee arthroplasty, Applied Bionics and Biomechanics 5 (2008), 77-87.

[36] J.G. Skedros, M.R. Dayton, C.L. Sybrowsky, R.D. Bloebaum and K.N. Bachus, Are uniform regional safety factors an objective of adaptive modeling/remodeling in cortical bone? Journal of Experimental Biology 206 (2003), 2431-2439.

[37] J.G. Skedros, M.W. Mason and R.D. Bloebaum, Modeling and remodeling in a developing artiodactyl calcaneus: A model for evaluating Frost's mechanostat hypothesis and its 
corollaries, Anatomical Record-Advances in Integrative Anatomy and Evolutionary Biology 263 (2001), 167-185.

[38] J. Stolk, N. Verdonschot, L. Cristofolini, A. Toni and R. Huiskes, Finite element and experimental models of cemented hip joint reconstructions can produce similar bone and cement strains in pre-clinical tests, Journal of Biomechanics 35 (2002), 499-510.

[39] F. Taddei, M. Viceconti, M. Manfrini and A. Toni, Mechanical strength of a femoral reconstruction in paediatric oncology: A finite element study, Proceedings of the Institution of Mechanical Engineers Part H-Journal of Engineering in Medicine 217 (2003), 111-119.

[40] M. Taylor, Finite element analysis of the resurfaced femoral head, Proceedings of the Institution of Mechanical Engineers Part H-Journal of Engineering in Medicine 220 (2006), 289-297.

[41] M.E. Taylor, K.E. Tanner, M.A.R. Freeman and A.L. Yettram, Stress and strain distribution within the intact femur: Compression or bending? Medical Engineering and Physics 18 1996), 122-131.

[42] W.R. Taylor, E. Roland, H. Ploeg, D. Hertig, R. Klabunde, M.D. Warner, M.C. Hobatho, L. Rakotomanana and S.E. Clift, Determination of orthotropic bone elastic constants using FEA and modal analysis, Journal of Biomechanics 35 (2002), 767-773.

[43] C.H. Turner, S.C. Cowin, J.Y. Rho, R.B. Ashman and J.C. Rice, The fabric dependence of the orthotropic elastic constants of cancellous bone, Journal of Biomechanics 23 (1990), 549-561.

[44] L. van Langendonck, J. Lefevre, A.L. Claessens, M. Thomis, R. Philippaerts, K. Delvaux, R. Lysens, R. Renson, B. Vanreusel, B. Vanden-Eynde, J. Dequeker and G. Beunen, Influence of participation in high-impact sports during adolescence and adulthood on bone mineral density in middle-aged men: A 27-year follow-up study, American Journal of Epidemiology 158 (2003), 525-533.

[45] M. Viceconti, L. Bellingeri, L. Cristofolini and A. Toni, A comparative study on different methods of automatic mesh generation of human femurs, Medical Engineering and Physics 20 (1998), 1-10.

[46] W.A. Weijs and B. Hillen, Cross-sectional areas and estimated intrinsic strength of the human jaw muscles, Acta Morphologica Neerlando-Scandinavica 23 (1985), 267-274.

[47] D.C. Wirtz, T. Pandorf, F. Portheine, K. Radermacher, N. Schiffers, A. Prescher, D. Weichert and F.U. Niethard, Concept and development of an orthotropic FE model of the proximal femur, Journal of Biomechanics 36 (2003), 289-293.

[48] R.C. Woledge, N.A. Curtin and E. Homsher, Energetic Aspects of Muscle Contraction, Academic Press, London, 1985.

[49] J. Wolff, Das Gesetz der Transformation der Knochen. Hirschwald, Berlin (Translated as The Law of Bone Remodeling by Maquet P., Furlong R., Springer, Berlin, 1986), 1892. 

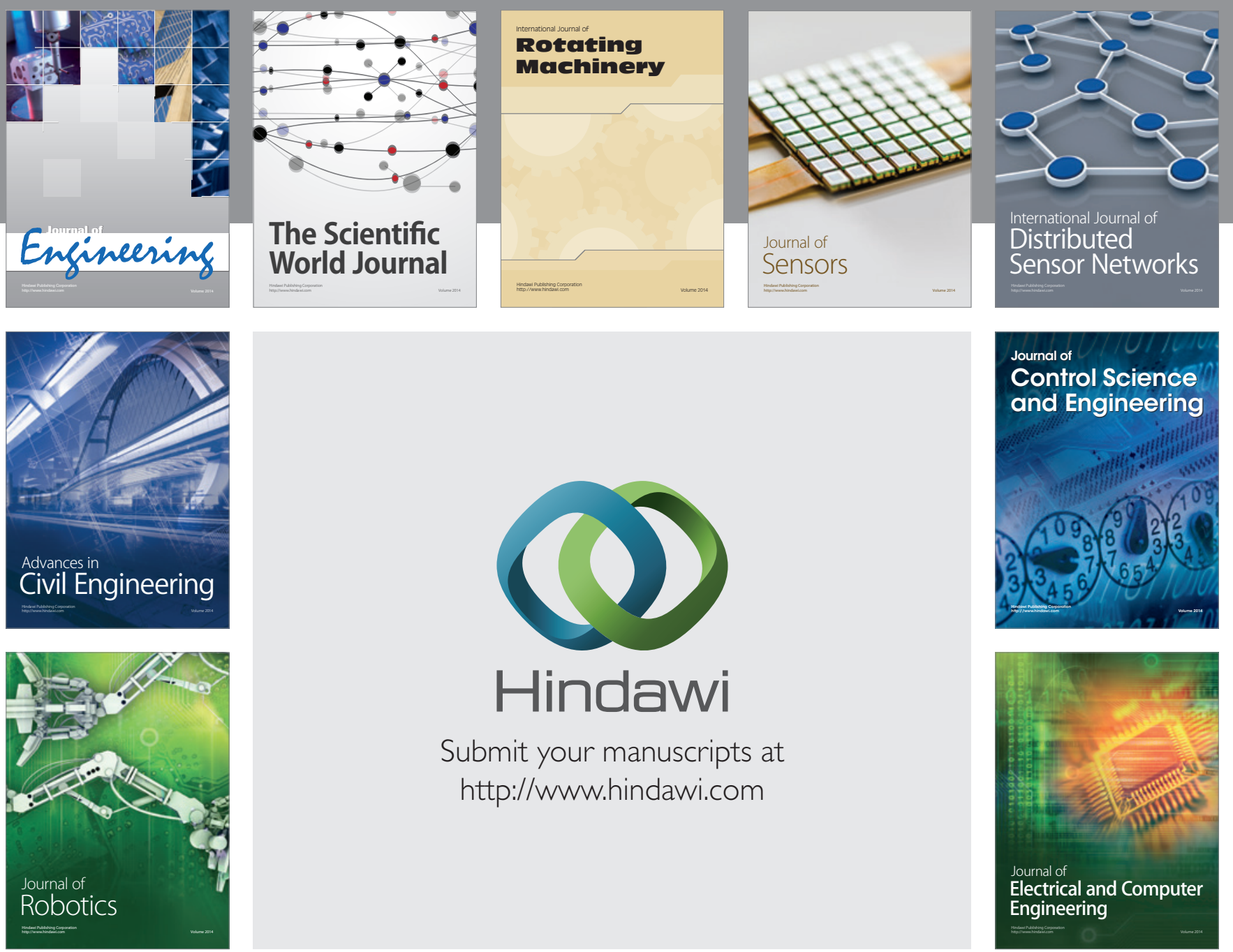

Submit your manuscripts at

http://www.hindawi.com
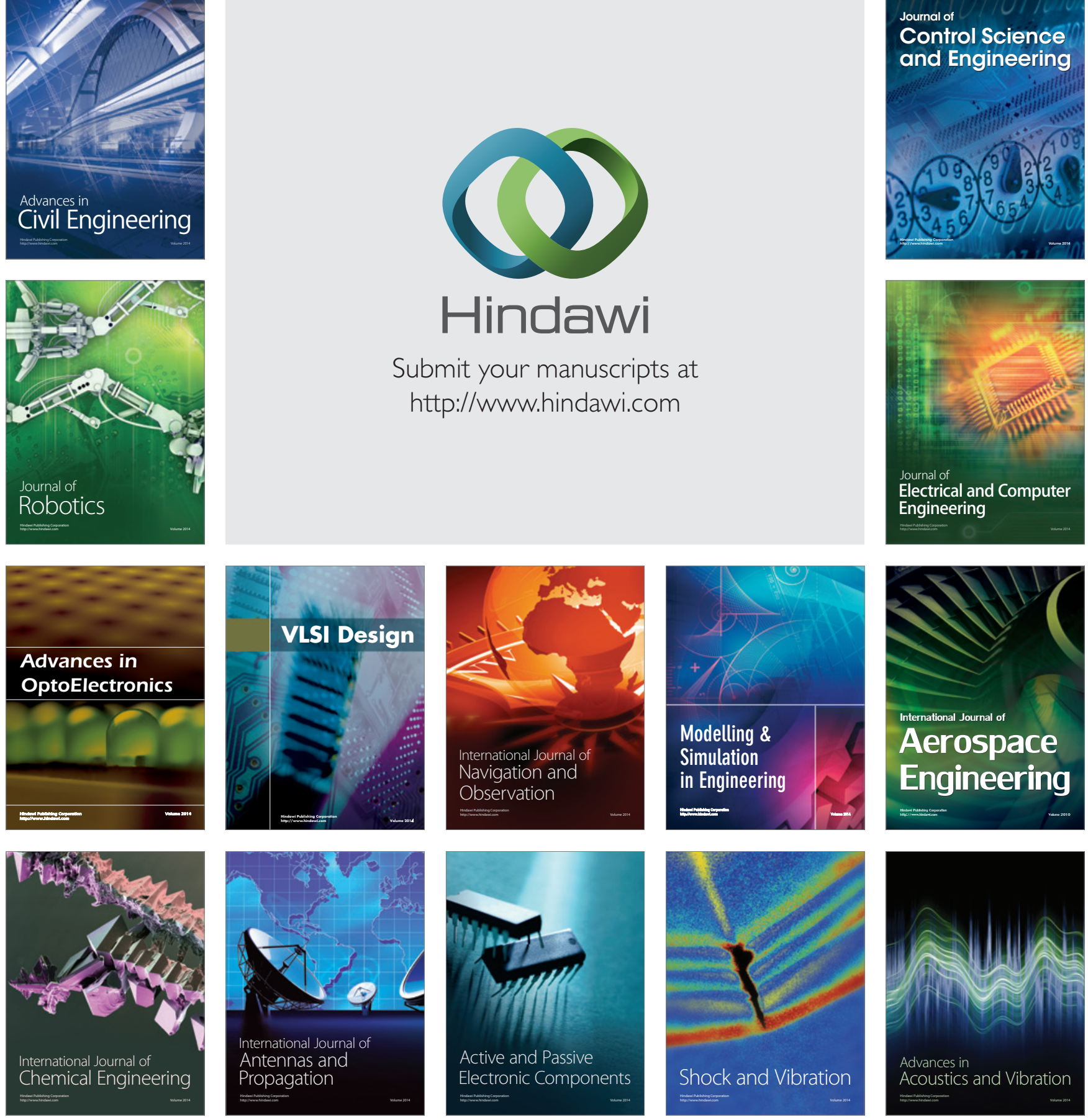